\title{
Surface Characterization of Colloidal Silica Nanoparticles by Second Harmonic Scattering: Quantifying the Surface Potential and Interfacial Water Order
}

Arianna Marchioro, ${ }^{1}$ Marie Bischoff, ${ }^{1}$ Cornelis Lütgebaucks, ${ }^{1}$ Denys Biriukov, ${ }^{2}$ Milan Předota, ${ }^{2}$ and Sylvie Roke ${ }^{1 *}$

${ }^{1}$ Laboratory for fundamental BioPhotonics (LBP), Institute of Bioengineering (IBI), and Institute of Materials Science (IMX), School of Engineering (STI), Ecole Polytechnique Fédérale de Lausanne (EPFL), CH-1015 Lausanne, Switzerland, . "Email: sylvie.roke@epfl.ch

${ }^{2}$ Institute of Physics, Faculty of Science, University of South Bohemia, 37005 České Budějovice, Czech Republic

Shorthand notations for independent tensor components

Table S1: Overview of the shorthand notation for independent tensor components used in this work.

\begin{tabular}{|c|c|}
\hline Indexed component & Tensor component \\
\hline$\chi_{1}^{(2)}$ & $\chi_{\perp \Perp}^{(2)}-\chi_{\|\| \perp}^{(2)}-\chi_{\|\perp\|}^{(2)}-\chi_{\perp\|\|}^{(2)}$ \\
$\chi_{2}^{(2)}$ & $\chi_{\|\| \perp}^{(2)}$ \\
$\chi_{3}^{(2)}$ & $\chi_{\|\perp\|}^{(2)}$ \\
$\chi_{4}^{(2)}$ & $\chi_{\perp\|\|}^{(2)}$ \\
\hline
\end{tabular}


Parameters used for surface potential fitting

Table S2: Parameters used for the AR-SHS fits of $\mathrm{NaOH}$ series

\begin{tabular}{|c|c|c|c|}
\hline $\mathrm{NaOH}$ addition & pH 5.7 & pH 10 & pH 11 \\
\hline Radius (nm) & 145 & 148 & 142 \\
\hline $\begin{array}{l}\text { Second harmonic wavelength } \\
(\mathrm{nm})\end{array}$ & 515 & 515 & 515 \\
\hline $\begin{array}{l}\text { Refractive index of the particle } \\
\text { (silica) }\end{array}$ & 1.46 & 1.46 & 1.46 \\
\hline $\begin{array}{l}\text { Refractive index of the solvent } \\
\text { (water) }\end{array}$ & 1.33 & 1.33 & 1.33 \\
\hline Temperature $\left({ }^{\circ} \mathrm{C}\right)$ & 23 & 23 & 23 \\
\hline Number of particles (particles $/ \mathrm{ml}$ ) & $3.50 \cdot 10^{10}$ & $3.50 \cdot 10^{10}$ & $3.50 \cdot 10^{10}$ \\
\hline Concentration of ions (M) & $4.02 \cdot 10^{-6}$ & 0.0001 & 0.001 \\
\hline
\end{tabular}

Table S3: Parameters used for the AR-SHS fits of $\mathrm{NaCl}$ series

\begin{tabular}{|c|c|c|c|c|}
\hline $\mathrm{NaCl}$ addition & pH 10 & $\begin{array}{c}\mathrm{pH} 10 \\
+0.1 \mathrm{mM} \\
\mathrm{NaCl}\end{array}$ & $\begin{array}{c}\mathrm{pH} 10 \\
+1 \mathrm{mM} \\
\mathrm{NaCl} \\
\end{array}$ & $\begin{array}{c}\mathrm{pH} 10 \\
+10 \mathrm{mM} \\
\mathrm{NaCl}\end{array}$ \\
\hline Radius (nm) & 147 & 146 & 142 & 143 \\
\hline $\begin{array}{l}\text { Second harmonic wavelength } \\
(\mathrm{nm})\end{array}$ & 515 & 515 & 515 & 515 \\
\hline $\begin{array}{l}\text { Refractive index of the particle } \\
\text { (silica) }\end{array}$ & 1.46 & 1.46 & 1.46 & 1.46 \\
\hline $\begin{array}{l}\text { Refractive index of the solvent } \\
\text { (water) }\end{array}$ & 1.33 & 1.33 & 1.33 & 1.33 \\
\hline Temperature $\left({ }^{\circ} \mathrm{C}\right)$ & 23 & 23 & 23 & 23 \\
\hline Number of particles (particles/ml) & $3.50 \cdot 10^{10}$ & $3.50 \cdot 10^{10}$ & $3.50 \cdot 10^{10}$ & $3.50 \cdot 10^{10}$ \\
\hline Concentration of ions $(\mathrm{M})$ & $1 \cdot 10^{-4}$ & $2 \cdot 10^{-4}$ & 0.0011 & 0.0101 \\
\hline
\end{tabular}

